\title{
An Empirical Research of Food Supply Chain Disruption Prevention
}

\author{
Hongliang Wang ${ }^{1,2,3}$ \\ ${ }^{1}$ School of Administration and Business, Zhengzhou University of Aeronautics \\ ${ }^{2}$ Henan Cooperative Innovation Center For Aviation Economic Development \\ ${ }^{3}$ School of economics, Huazhong University of Science and Technology, Wuhan, Hubei, \\ 430074,Zhengzhou China \\ Yes2001@zzia.edu.cn
}

Keywords: Food emergencies; Food supply chain; Risk; Game model

\begin{abstract}
Food emergencies happened frequently, Which has led people to strong concern for the quality of food, while the quality of food supply chain has a decisive impact on the final products. Therefore, security risk monitoring on food supply chain must be more stringent. Strengthening the supervision of cheating behaviors is a necessary way to prevent food emergencies. The paper sets up a game model about food quality behaviors in a food supply chain which contains one core enterprise and a few cooperators, and the core enterprise play a dominant role in the food supply chain. This paper studies effect of the two supervision ways implemented by core enterprise, and also contrasts their effects in prevention food emergencies.
\end{abstract}

\section{Introduction}

Food is one of people life's necessities, related to people's survival and health, and people pay close attention to food. The "poisoned dumplings" affair took place in 2008 between China and Japanese, even though the main reason whether lies in China's production process or lies in the Japanese terminal tetailers pollution problems, still has no conclusion. It has given the Chinese food factory in Hebei province a disastrous blow, which suffered serious losses. Which has led people to strong concern for the quality of food, while the quality of food supply chain has a decisive impact on the final products. Therefore, security risk monitoring on quick- food supply chain must be more stringent. Safety and quality control is necessary on the entire supply chain from material origin to the consumers.

However, from a deep study of food emergencies in recent years, the vast majority of food emergencies came from food quality cheating behaviors in the process of food production, delivery and sale, thus causing the food supply chain risks continue to accumulate and eventually the food emergency broke out outbreak. Therefore, from the perspective of food supply chain to research food quality supervision mechanism is an effect way to prevent and eliminate food emergencies. In most food emergencies, the core enterprise in a food supply chain often suffers the biggest loss. Therefore, the core enterprise are willing and capable to supervise the cooperator`s food quality to ensure the food supply chain running smoothly and health. Based on considerations above, this paper studies a food supply chain which consists of a core business and a few cooperators, and sets up a game model to demonstrate the food quality behaviors between core enterprise and its cooperators [1]. In the game model, the core enterprise plays a dominant role and its cooperators acts as subordinate roles. The core enterprise supervises the cooperator's food quality by the ways of cheating behaviors detecting and severe punishment of cheating behaviors. Correspondingly, the cooperators can choose honest or cheating behavior to maximize its own earnings.

The paper is conducted to find out the effect of the two supervision ways implemented by core enterprise, and also contrasts their efffects in prevention food emergencies. 


\section{The Influence of Food Quality Supervision upon Food Supply Chain Disruption}

The food supply chain is a network of corporate alliances which consists of raw material suppliers, food manufacturers, distributors and retailers. The food quality is affected by each member in the food supply chain, and any cheating behaviors in food quality by cooperators will cause risk or even food emergencies. We take the "poisoned dumplings" affair took place in 2008 between China and Japanese as an example to illustrate, which as shown in Fig. 1),

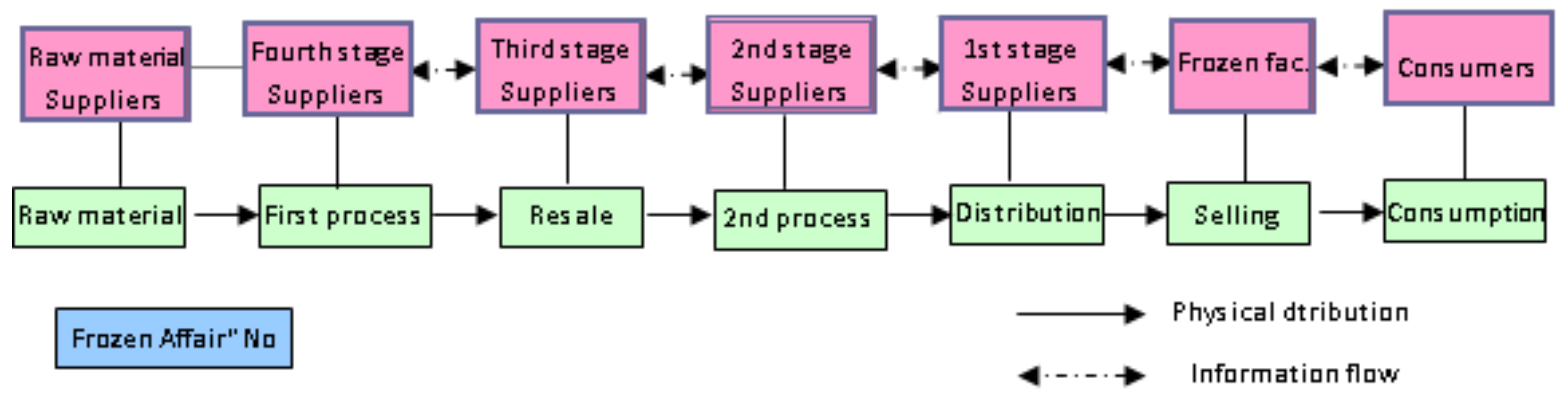

Figure 1 The linearstructure af Quick frazen fand s upply chain

In recent years, food emergencies happen frequently. The deep reasons may conclude two aspects, and the first reason is related with the characteristics of the food. Food quality is concealed and usually difficult to identify by naked eyes, but its harmful effect to human bodies is direct. The second reason is related with food supply chain management. For many food supply chains, the cooperator`s food quality promise have not legal contracts to guarantee, and just depend on long-term relationship or trust between each other. Once the food emergencies occur, the smaller raw material suppliers and vendors often suffer limited losses. This will contrast efffects of the two ways above in prevention food emergencies.

Influence of Detection Probability upon Food Emergencies. In this paper, detection probability can be defined as the probability that the cooperator`s cheating behaviors can be detected by the core enterprise in the food supply chain. In theory, detection probability is affected by supervision, honest or dishonest earnings, trading information and trading systems. If the core enterprise implement greater supervision upon cooperator`s food quality behaviors, the detection probability of cheating behaviors will be higher. Correspondingly, the supervision cost will be higher. If cooperators can gain more earnings from cheating behaviors than honest behaviors, they will be more likely to cheat and conceal their cheating behaviors, so the detection probability will decrease. The more transparent of transaction information on both sides, the higher the detection probability will be. In addition, if the trading system supplies rewards for disclosure cheating behaviors or good credits kept by cooperative enterprises, it can improve the detection probability.

Detection probability has deterrent forces on cooperator`s food quality behaviors in the food supply chain, and its mechanism can be demonstrated game model between the core enterprise and its cooperators. The game process can be described as followings: the core business and its member enterprises of the game process can be simply described as: the core enterprise has two choices. when it implement lower supervision with lower cost, then gain a lower detection probability when it implement greater supervision with higher cost, then gain a higher detection probability. Once the core enterprise finds out cheating behaviors, it will give severe penalties. The cooperative enterprises are rational, they aims to maximize their own earnings by two choices, when the detection probability is lower, they will carry out cheating behaviors and food supply chain risk increases. But if detection probability is higher, they will carry out honest behaviors and food supply chain risk decreases. Correspondingly, the food emergencies reduce.

Some Hypotheses Concerning the Conditions of Game Model.

The hypotheses concerning the condition of game model are as follows: 
(1) The food supply chain comprises one core enterprise and a few cooperators, and they all want to maximize its own earnings.

(2) Suppose the game model is a static game model. In other words, when considering the strategies, core enterprise and cooperators will regard their counterpart's strategy as a fixed one without thinking their choice's influence upon other's;

(3) Each participant has complete information about his counterpart, including character, strategy space and payoffs function;

(4) No disturbance from the third parties;

The Foundation of the Game Model and the Result Analysis. According to the hypothesis above, we set up a game model between core enterprise and cooperators. In this model, we just focus on the behavior choices and earnings of cooperators. The concrete conditions are shown in the fig 1[2-3].

According to fig. 1, the expected earnings of cooperators acquires can be written as:

$U=r *[P * R+(1-P) * R]+(1-r)[P(-T)+(1-P) * e]$

Where

$P$ is the high detection probability, and $(1-P)$ is the low detection probability. The value of $P$ rises as the investment becomes higher;

$r$ is the probability that cooperators deceives; and $(1-r)$ is the honest behaviors probability;

$R$ is the market average earnings that all cooperators acquires in keeping honest behaviors;

$e$ is the earnings that the cooperators acquires when he cheat and succeeds;

$T$ is the penalty that cheating behaviors are fined when detected;

$U$ is the final proceeds that the cooperators acquires in the game.

The equation (1) can also be written as:

$$
r=[U+(P * T+e * P-e)] \div[R+(P * T+e * P-e)]
$$

Define $k=P * T+e * P-e$, then:

$$
r=(U+k) /(R+k)
$$

And $\quad k=P(T+e)-e$

From the equation (4), $k$ is positively correlated with $P$ and $T$. In other words, $k$ rises when $P$ or $T$ increases.

According to the hypothesis (1), the cooperators are rational.

So, $U \geq R$

Therefore, $r$ is negatively correlated with $k$ in the equation (3).

In a word, $r$ is negatively correlated with $P$ and $T$, which also means $r$ decreases when $P$ or $T$ increase respectively or together.

From the demonstration above, we can draw a conclusion, namely, the cooperators will make a choice between honest and cheating behaviors, in order to get the expected earnings. However, this choice is restricted by the detection probability. The cheating behaviors probability $(r)$ will descend so as to get the expected earnings ( $U$ ), as the core enterprise's investment increases and the detection probability $(P)$ rises accordingly. On the contrary, the cheating behaviors probability $(r)$ will climb 
up so as to get the expected earnings ( $U$ ), as the core enterprise's investment decreases and the detection probability $(P)$ falls accordingly. From the analysis above, the detection probability has a deterrent force on food emergencies.

Influence of Punishment Degree upon Food Emergencies. Besides the detection probability, there is another supervision way, namely punishment degree, and it has important influence on the cheating behaviors. The punishment degree is the magnitude that the core enterprise in the food supply chain punishes the cheating behaviors. Punishment degree has a direct influence on food emergencies and the mechanism is similar to the detection probability.

\section{A Comparison of Influence upon Food Emergencies from Detection Probability and Punishment Degree}

The Joint Effect of Detection Probability and Punishment Degree. We have studied the influence of detection probability and punishment degree respectively with the purpose to simplify the study. In fact, detection probability and punishment degree interacts with each other in practice.

In other words, the influence from the detection probability and punishment degree upon food supply chain risk is magnified and strengthened reciprocally. Therefore, the relationship between the mutual influence from detection probability and punishment degree and food supply chain risk can be described as following: for a given cheating behavior and fixed punishment degree, the higher the detection probability is and the lower of food supply chain will be; For a given cheating behavior and fixed detection probability, the higher the punishment degree is and the lower the food supply chain risk will be.

A Comparison of Influence upon Food Supply Chain Risk from Detection Probability and Punishment Degree. We can make a further study to find which supervision way is more effective between detection probability and punishment degree to a dishonest cooperator when his tolerance of expected penalty is definite. We can set up a simple model to solve this problem.

When the detection probability, penalty and the original income are defined as $P, T$ and $w$ respectively, the cooperators tend to be a risk averter and his expected earnings is as following:

$$
\bar{V}=E u(P, T)=P u(w-T)+(1-P) u(w)
$$

If $P$ and $T$ are replaced by $s P$ and $T / s(s>0)$ respectively, the expected earnings of the dishonest cooperator can be described as following:

$$
V(s)=E u(s P, T / s)
$$

$$
V(s)=s P u(w-T / s)+(1-s P) u(w)
$$

Obviously, $V(1)=\bar{V}$. The differential coefficient of (2) is as following:

$$
V^{\prime}(s)=P u(w-T / s)+\frac{T}{S} P u^{\prime}\left(w-\frac{T}{s}\right)-P u(w)
$$

By the theorem of Lagrange differential coefficient median, the right part of equation above equals: 


$$
-\frac{T}{S} u^{\prime}(w-\theta T / s)+\frac{T}{s} u^{\prime}\left(w-\frac{T}{s}\right)=-\left(\frac{T}{s}\right)^{2} u^{\prime \prime}(w-\mu T / s)(1-\theta)>0
$$

Where $\theta, \mu \in(0,1)$. The inequation above is set on the hypothesis that the trader is a risk averter $\left(u^{\prime \prime}<0\right)$.

Due to $V^{\prime}(s)>0, V(1)=\bar{V}$ and $s<1$, so $V(s)<\bar{V}$.

The result above means that the increase of punishment degree and the decrease of detection probability will promote the supervision effect in food supply chain risk management.

\section{Conclusion}

Through the theoretic study above, it's proved that food supply chain risk is an important source of food emergencies, and the supervision methods are effective ways to prevent food supply chain risk. However, the conclusion above comes from normative study and need to be tested in practice. Therefore, the related demonstration is the main direction of this field in future.

\section{Acknowledgements}

This work is supported by Soft science project of Henan provincial science and Technology Department, Project NO

\section{References}

[1] Henri Pascal Sanders, Lelievre Nathalie Cadieu. Implementation and assessment of the quality management system in research in a laboratory of the French Food Safety Agency: application to Phd student work.Accred qual assur ,2014) 14:207-217

[2] R.G.BATSON,K.D.MCGOUGH, A new direction in quality engineering: supply chain quality modeling, International Journal of Production Research, Vol.45, No.23, 1 December 2007,5455-5464.

[3] Xiande Zhao., Decision Sciences Research in China: Current Status, Opportunities, and Propositions for Research in Suply Chain Management, Logistics, and Quality Management. Decision Science., Vol.38.2007,P39-80.

[4] Kashi R.Balachandran, Suresh Radhakrishnan., Quality Implications of Warranties in a Supply Chain.., Management Science., VOL.51,No.8,August 2005, pp.1266-1277

[5] Zhang Weiying, Game theory and Economy Information Science, Shanghai: Shanghai Sanilac bookstore, Shanghai People's Publishing House, 1996 (in Chinese)

[6] Hongliang Wang. An Empirical Research on Quality and Safety of The Quick-Frozen Food Supply Chain. 2010 International Conference of Information Science . Xi' an, China, August 7 8, 2010 